\title{
The combination of brush cytology with the results of histopathological examination in laryngeal cancer diagnosis
}

\author{
Paulina Lepka ${ }^{1}$, Agnieszka Hałoń2, Szczepan Barnaś ${ }^{3}$, Tomasz Zatoński ${ }^{4}$
}

${ }^{1}$ Clinic of Otolaryngology and Head and Neck Surgery, 4th Military Clinical Hospital with Polyclinic in Wroclaw, Poland

${ }^{2}$ Department of Pathomorphology and Oncological Cytology, Wroclaw Medical University, Head of the Department, Poland

${ }^{3}$ Clinic of Otolaryngology and Head and Neck Surgery, 4th Military Clinical Hospital with Polyclinic in Wroclaw, Poland

Abstract $\quad{ }^{4}$ Department and Clinic of Otolaryngology and Head and Neck Surgery, Wroclaw Medical University, Wrocław, Poland

Recently, the incidence rate of head and neck cancer (HNC) has been increasing significantly. It is estimated that there are over 550,000 new cases per year, of which over 130,000 are laryngeal cancers. It is assumed that in more than $60 \%$ of patients the disease is diagnosed late, at stages III-IV, which is associated with unfavorable prognoses: the average survival ranges from $15 \%$ to $45 \%$. The mainstay of successful tumor therapy is the early detection of neoplastic tissue. The laryngological examination with the use of traditional instruments should be expanded with an endoscopic examination of the larynx using optics in the outpatient clinics. This procedure is sufficient to select patients who need a direct laryngoscopy with a surgical biopsy, usually under general anesthesia in operating room conditions. However, it may bear potential complications. In 1941, Papanicolaou and Traut showed that brush cytology could be useful in detecting precancerous conditions and cervical cancers. For decades, research on the usefulness of brush cytology in diagnosing precancerous conditions and laryngeal cancers has been conducted. This paper aims to enable the reader to understand the issues related to laryngeal cancer and present the results of the previous use of brush cytology in the diagnostic process.

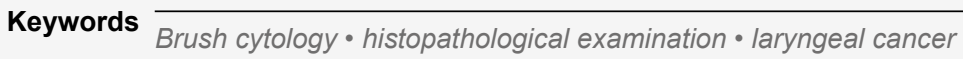

\section{Introduction}

According to the National Cancer Registry, the laryngeal cancer incidence in 2010 reached 2,200 cases $(1,900$ men and approx. 300 women), representing about $2.7 \%$ of malignancies in men and $0.4 \%$ in women. More than $90 \%$ of cases occur in patients over 50 ; the risk increases with age, reaching its peak in men over 70 and women at the turn of the fifth and sixth decade of life. The incidence of laryngeal cancers in men constantly increased until the mid-1990s, after which an incidence decline was observed; however, it remains stable in women. The 5 -year survival rate is $51 \%$ for men and $63 \%$ for women. In Poland, both the morbidity and mortality due to larynx cancer are higher than in other European Union countries [1, 2]. It is estimated that laryngeal cancer incidence in the United States of America in 2016 reached 13,430, with more than 3,620 deaths reported [3]. According to American statistics, it is one of the few oncological diseases for which the 5-year survival rate decreased over the last 40 years (from $66 \%$ to $63 \%$ ). However, the overall incidence rate is decreasing [3]. It is assumed that in more than $60 \%$ of patients, the disease is diagnosed at stages III-IV, which is associated with unfavorable prognoses: the average survival ranges from $15 \%$ to $45 \%[4,5]$. This reveals the need for further research and innovation in this area. The risk factors for laryngeal cancer development include alcohol consumption and smoking [6]. Smoking is linearly related to laryngeal cancer; the risk among smokers is 10-15 times higher than among non-smokers. The heaviest smokers have up to 30 times higher risk of developing this illness [7, 8]. HPV plays a significant role in the pathogenesis of cancer of the oral pharynx. In contrast, the impact of 
oncogenic HPV infections $(16,18,31,33,35)$ on the cancer transformation process in laryngeal malignancies appears to be much smaller and not fully understood. It is estimated that viral DNA incidence ranges from $20 \%$ to $30 \%$, but this percentage varies among researchers and depends on the detection method applied [9, 10]. The most prevalent is squamous cell carcinoma (SCC). SCCs are divided into well differentiated (G1), moderately differentiated (G2), poorly differentiated (G3), and anaplastic (G4) carcinomas, depending on keratosis and several miotic figures [11]. Lesions in epithelial cells precede the development of SCC, which determines the formation of dysplasia. Roegan introduced the term dysplasia in 1958; it refers to atypical proliferation and lesions in both cell and epithelial structures $[12,13]$. Leucoplakia, erythroplakia, and epithelial ulceration are clinical diagnoses that raise suspicions of neoplastic transformation; in histopathological diagnosis, they indicate dysplasia at various stages, carcinoma in situ, or invasive cancer [14]. In dysplasia, the neoplastic transformation grade is about $36.4 \%$ and represents a relatively late multistage carcinogenesis phase [15]. Forecasting of the transformation potential depends on the histological evaluation, which involves the quantitative determination of the degree of epithelial architecture abnormalities and cellular ones above the basal membrane [14]. Such symptoms as hoarseness, weakening of the voice, dysphagia, dyspnea, neck tumors, or ear pain should lead to further diagnostic processes for laryngeal cancer. The diagnosis should be based on a physical examination, taking risk factors into account, and on a laryngological analysis using a nasofiberscope. An optical technique, narrow band imaging (NBI), based on the modification of white light using special filters, is instrumental in detecting the microvascular abnormalities associated with the preneoplastic and neoplastic changes in the mucosal lining. This procedure can be performed as a diagnostic and during endoscopic laser cordectomy, as it allows a more precise assessment of the status of surgical margins of early-stage and locally advanced laryngeal cancers $[16,17]$. Narrow band imaging is also a practical tool in laryngeal cancer follow-up after surgical procedures such as transoral laser microsurgery or radiotherapy [18]. This diagnostic process is not always sufficient; however, it can be supplemented with direct laryngoscopy using the collection of samples for histopathological examination, usually performed under general endotracheal anesthesia in operating room conditions. In cases of general anesthesia contraindications or cervical spine alterations, an alternative procedure should be applied. Videolaryngoscopy during conscious sedation might be considered as a surgical approach in patients who are not suitable for phonosurgery by microlaryngoscopy. It is essential to mention that during surgical biopsy of the larynx under local anesthesia, unexpected bleeding or laryngospasm may occur [19].

After receiving the histopathological diagnosis, it is necessary to evaluate the extent of the disease-that is, the disease stage at the time of diagnosis. Standard imaging techniques include computed tomography (CT), magnetic resonance imaging (MRI), and positron emission tomography (PET). The advantage of CT over MRI for laryngeal cancer is debatable; CT usually shows a higher specificity in the identification of thyroid cartilage invasion compared to MRI [20]. The disease stage at the time of diagnosis is a critical predictive factor. It is estimated that the T3-T4 stage patients are twice as likely to die compared to those at an earlier stage; the 5-year survival rate for $\mathrm{T} 1$ is about $95.6 \%$, while for T4 it is only $52.1 \%$ [21]. Therefore, the effective diagnostic process leading to early-stage laryngeal cancer diagnosis through histopathological examination is of great importance. The histopathological examination is a gold standard in laryngeal SCC diagnosis. However, it is difficult to monitor the effects of treatment or conduct the follow-up of a patient with low- or high-grade dysplasia by performing regular laryngeal microsurgeries, which, in addition to tissue traumatization, carry the risk of infection or perioperative bleeding. Moreover, the follow-up of a patient with a history of laryngeal cancer is essential, due to the high rate of relapse and the frequent occurrence of a second primary cancer of this area, which is a result of the negative effect of carcinogenic factors on the entire mucous membrane of the upper digestive and respiratory tract [22, 23]. To minimalize the risks associated with surgery under general anesthesia, other auxiliary procedures may be considered. In both gynecology and pulmonology, brush cytology is an additional tool for diagnosing and monitoring cervical, lung, and bronchial cancers [24].

\section{Material and methods}

This review aims to introduce the contribution of exfoliative cytology to the diagnosis of precancerous conditions and laryngeal cancers. It considers the following question: "Is brush cytology a highly reliable and valuable diagnostic procedure for clinically suspected laryngeal lesions?"

A comprehensive and detailed literature search was conducted to answer the above question. The abstracts of the studies identified in the search were screened, and subsequently, full texts were selected. The primary source of literature included search engines and online databases with articles regarding medicine and life science, such as PubMed and Google Scholar. The other source of data was the Wroclaw Medical University library service. 
Keywords or phrases used in the research included brush cytology, exfoliative cytology of larynx, and otolaryngology, combined with cytology and histopathology, squamous cell carcinoma of the larynx. The included criteria combined the use of histopathology with touch smear cytology in suspected malignant lesions of the larynx. The studies concerning only benign diseases were excluded. As a result, 11 relevant articles were identified across 66 years, from 1951 to 2017.

\section{Discussion}

In 1941, Papanicolaou and Traut showed that brush cytology could be useful in detecting precancerous conditions and cervical cancers [12]. The role of brush cytology in the diagnosis of laryngeal diseases has been explored for years. It appears that Morrison et al. were the first to present the results of the study (1964), which compared cytology and histopathology results in terms of laryngeal cancer - 8 out of 9 compared results were consistent [25]. In 1951, Friedmann compared brush cytology and histopathology results in 212 patients with suspected malignant lesions within the head and neck, 106 of whom were patients with suspected laryngeal cancer. The clinical diagnosis was confirmed histopathologically in 110 cases and cytologically in 105 cases. Friedmann also raised the importance of brush cytology for laryngeal diseases, concluding that laryngeal swabs have fewer non-specific cells than nasal, nasopharyngeal, or sinus ones [26]. In 1968, W. F. Frable and M. A. Frable obtained a positive result from brush cytology in 14 out of 19 laryngeal cancer cases. The authors also emphasize that the presence of hyperkeratosis in a brush smear should raise suspicion of SCC cells [27]. Glennie et al. (1976) obtained full compliance in cytological and histopathological diagnoses in benign lesions of the larynx. In malignancies, the result was consistent in 20 out of 22 cases [28]. In the early 1980s, in their prospective study involving 350 patients, Lundgren et al. achieved brush cytology sensitivity and specificity of $83 \%$ and $84 \%$ to detect moderate- and high-grade dysplasia, carcinoma in situ, or invasive cancer. The authors noted that false-negative swab results were found in patients who previously underwent radiotherapy of the head and neck region or were diagnosed with moderate-grade dysplasia. In this study, a positive result was obtained in the smear, whereas the surgical biopsy was negative. The result was confirmed by the next surgical biopsy [29]. In 1994, Waloryszak and Makowska, willing to evaluate the usefulness of brushing smears in detecting laryngeal cancer, examined 70 patients, finding consistent results in $91 \%$ of cases; the remaining $9 \%$ were false-negative results [30]. In 2000, Malamou-Mitsi et al. obtained $100 \%$ specificity of brush cytology in a similar study involving a group of 30 patients, without obtaining false-positive results and with a sensitivity of more than $93 \%$, with one false-negative result of smear for non-Hodgkin's lymphoma of the larynx [31]. In 2006, Ustundag et al., analyzing the results of 135 patients, obtained sensitivity and specificity of brush cytology of the order of $82.5 \%$ and $94.5 \%$ in the diagnosis of benign and malignant laryngeal lesions [32]. In 2006, Gugatschka et al. recruited 130 patients who underwent laryngostroboscopic examination before microsurgery of the larynx with the brushing smear collection. The results of the study showed the sensitivity of cytology as an isolated test in the range of $74 \%$, while the combination of this diagnostic method with laryngostroboscopic examination made it possible to obtain over $97 \%$ sensitivity in the detection of glottic cancer [33]. In 2015, in their study involving 30 patients, Chatziavramidis et al. achieved $85 \%$ sensitivity and over $90 \%$ specificity using restrictive exclusion criteria. The study group did not include patients with radio- or chemotherapy for HNC nor patients with chronic cardiovascular and lung diseases. Brush cytology, liquid-based cytology, and surgical biopsy were performed. Additionally, VEGF expression was determined in the material from liquid-based cytology. The high VEGF expression is associated with a higher relapse rate and faster disease progression [34]. In the framework of the 2017 research conducted by Ajayan et al. involving a group of 50 patients, slightly over $70 \%$ sensitivity of brush cytology and $100 \%$ specificity were obtained. The sensitivity of brush cytology was significantly higher in the diagnosis of poorly differentiated squamous cell carcinomas (SCCs) [35]. In many cases, directly following the administration of anesthesia and applying a Kleinsasser laryngoscope, a brush smear was first performed, and then the histopathological examination sample was collected from the same region $[25,29,31,35]$. Ustundag applied the reverse order in his study by first collecting the histopathological sample and then performing a brushing smear from the collected sample [32]. Despite differences in smear sample collection, the results reported by Ustundag do not differ significantly from others. In most cases, the sample collected during brush cytology was applied to a glass slide, fixed in $95 \%$ ethyl alcohol, and stained using Papanicolaou [25, 29, 31, 35] or May-Grünwald-Giemsa staining techniques [32]. Despite different staining techniques, Ustundag received comparable results.

The authors agree that brush cytology can be a useful auxiliary examination in the diagnostic process of precancerous conditions and laryngeal cancers. Lundgren et al. emphasize that a positive result of the brush cytology together with a negative biopsy result prompts the performance of a repeat biopsy. In contrast, a negative cytology result does not exclude cancer. Lundgren also noted that brush cytology results are less reliable in patients after 
radiotherapy or with moderate-grade dysplasia [29]. On the other hand, Malamou-Mitsi et al. claim that brush cytology can be used as a stand-alone diagnostic method where a surgical biopsy is either contraindicated or impossible or frequently repeated biopsies can adversely affect the larynx structure and function [31]. Ustundag et al. state that the best diagnostic method combines histopathological and cytological examination [32]. At the same time, Gugatschka et al. highlight the role of stroboscopic examination, which in combination with brush cytology can be both a satisfactory screening test for detecting early glottic cancer and useful for long-term patient follow-up [33]. Ajayan et al. stress that brush cytology is a cheap and minimally invasive examination that provides a quick, simple, and reliable result and should be widely used in the diagnostic process of precancerous conditions and laryngeal cancers. Nevertheless histopathological examination remains the gold standard in making the diagnosis [35].

\section{Conclusion}

The results of brush cytology in detecting precancerous conditions and laryngeal cancers are ambiguous and differ in studies. Its sensitivity ranges from $70 \%$ to $93 \%$; however, it usually reaches approximately $80 \%$ and can be increased to almost $97 \%$ when combined with another diagnostic tool such as laryngostroboscopy. Moreover, false-negative swab results are found in patients who previously underwent radiotherapy of the head and neck region. Therefore, brush cytology may not be a suitable diagnostic tool for all patients. It is necessary to underline that exfoliative cytology and histology are not competitive diagnostic procedures. Histopathology examination of laryngeal precancerous conditions and malignancy is a gold standard. Furthermore, brush cytology appears to be a very cheap, simple, costeffective, and relatively accurate test that gives a speedy diagnosis. It may be performed under local anesthesia, and even consecutive cytology procedures do not lead to vocal folds, scars, or a voice impairment, which may occur after subsequent biopsies. To summarize, exfoliative cytology is not suitable for a final diagnosis, but it may be included as an additional tool in the early diagnostic process at the prehospitalization stage.

\section{Abbreviations}

CT - computed tomography, HPV - human papillomavirus, MRI - magnetic resonance imaging, PET - positron emission tomography, SCC - squamous cell carcinoma, VEGF vascular endothelial growth factor.

\section{Authors' Contribution}

P.L.: reserch concept and design, acquisition of data, data analysis and interpretation, writing: original draft preparation, visualization, literature review; A.H.: supervising the project, data analysis and interpretation, writing: review and editing, final proofreading, and approval of the version for publication; S.B.: supervising the project, visualization, literature review, final proofreading and approval of the version for publication; T.Z.: supervising the project, data analysis and interpretation, writing: review and editing, final proofreading, and approval of the version for publication

\section{Conflict of Interest}

The authors have no potential conflicts of interest to declare.

\section{References}

[1] Jemal A., Bray F., Center M.M., Ferlay J., Ward E., Forman D.: Global cancer statistics. CA Cancer J. Clin., 2011; 61: 69-90.

[2] Krajowy Rejestr Nowotworów [Polish National Cancer Registry]. http://www.onkologia.org.pl (28.10.2019).

[3] Siegel R.L., Miller K.D., Jemal A.: Cancer statistics, 2016. CA. Cancer J. Clin,. 2016; 66, 7-30.

[4] Chan J.Y., Wei W.I.: Current management strategy of hypopharyngeal carcinoma. Auris Nasus Larynx, 2013; 40: 2-6.

[5] Groome P.A., O'Sullivan B., Irish J.C., Rothwell D.M., Schulze K., Warde P.R., Schneider K.M., Mackenzie R.G., Hodson D.I., Hammond J.A., Gulavita S.P., Eapen L.J., Dixon P.F., Bissett R.J., Mackillop W.J.: Management and outcome differences in supraglottic cancer between Ontario, Canada, and the surveillance, epidemiology, and end results areas of the United States. J. Clin. Oncol., 2003; 21: 496-505.

[6] Hoffman H.T., Porter K., Karnell L.H., Cooper J.S., Weber R.S., Langer C.J., Ang K.K., Gay G., Stewart A., Robinson R.A.: Laryngeal cancer in the United States: Changes in demographics, patterns of care, and survival. Laryngoscope, 2006; 116: 1-13.

[7] Kuper H., Boffetta P., Adami H.O.: Tobacco use and cancer causation: Association by tumour type. J. Intern. Med. 2002; 252: 206-224.

[8] Rothman K.J., Cann C.I., Flanders D., Fried M.P.: Epidemiology of laryngeal cancer. Epidemiol. Rev.1980; 2: 195-209.

[9] Gama R.R., Carvalho A.L., Filho A.L., Scorsato A.P., Mendoza López R.V., Rautava J., Syrjänen S., Syrjänen K.: Detection of human papillomavirus in laryngeal squamous cell carcinoma: Systematic review and meta-analysis. Laryngoscope, 2016; 126 : 885-893.

[10] Ndiaye C., Mena M., Alemany L., Arbyn M., Castellsagué X., Laporte L., Bosch F.X., de Sanjosé S., Trottier H.: HPV DNA, E6/ 
E7 mRNA, and p16INK4a detection in head and neck cancers: A systematic review and meta-analysis. Lancet Oncol., 2014; 15: 1319-1331.

[11] Almamangush A., Mäkitie A.A., Triantafyllou A., de Bree R., Strojan P., Rinaldo A., Hernandez-Prera J.C., Suárez C., Kowalski L.P., Ferlito A., Leivo I: Staging and grading of oral squamous cell carcinoma: An update. Oral Oncol., 2020; 107: 104799.

[12] Lumerman H., Freedman P., Kerpel S.: Oral epithelial dysplasia and the development of invasive squamous cell carcinoma. Oral Surg. Oral Med. Oral Pathol. Oral Radiol. Endod., 1995; 79: 321-329.

[13] Zerdoner D.: The Ljubljana classification - its application to grading oral epithelial hyperplasia. J. Craniomaxillofac. Surg., 2003; 31: 75-79.

[14] Sadiq H., Gupta P., Singh N., Thakar S.S., Prabhakar I., Thakral J.: Various grading systems of the oral epithelial dysplasia: A review. Int. J. Adv. Health Sci., 2015; 1: 20-26.

[15] Fischer D.J., Epstein J.B., Morton T.H., Schwartz S.M.: Interobserver reliability in the histopathologic diagnosis of oral pre-malignant and malignant lesions. J. Oral Pathol. Med., 2004; 33: 65-70.

[16] Kimza H., Jackowska J., Wierzbicka M.: The usfulness of the NBI - narrow band imaging for the larynx assessment. Otolaryngol. Pol., 2018; 72: 1-3.

[17] Piazza C., Del Bon F., Peretti G., Nicolai P.: Narrow band imaging in endoscopic evaluation of the larynx. Curr. Opin. Otolaryngol. Head Neck Surg., 2012; 20: 472-476.

[18] Witkiewicz J., Klimza H., Piersiała K., Jackowska J., Wierzbicka $\mathrm{M}$.: The usefulness of the narrow band imaging (NBI) in decisionmaking process regarding second look procedure $(\mathrm{SL})$ in laryngeal cancer follow-up after transoral laser microsurgery. PLoS One, 2020; 15: e0236623.

[19] Saita V., Allegra E., Marino N., Trapasso S., Monea M.C.: Videolaryngoscopy during conscious sedation in patients not suitable for phonosurgery by microlaryngoscopy: A pilot study. ORL J. Otorinolaryngol Relat. Spec., 2017; 79: 185-190.

[20] Chu E.A., Kim Y.J.: Laryngeal cancer: Diagnosis and preoperative work-up. Otolaryngol. Clin. North Am., 2008; 41: 673-695.

[21] Ramroth H., Schoeps A., Rudolph E., Dyckhoff G., Plinkert P., Lippert B., Feist K., Delank K.W., Scheuermann K., Baier G., Ott I., Chenouda S., Becher H., Dietz A.: Factors predicting survival after diagnosis of laryngeal cancer. Oral Oncol., 2011; 47: 1154-1158.

[22] Leemans C.R., Braakhuis B.J., Brakenhoff R.H.: The molecular biology of head and neck cancer. Nat. Rev. Cancer, 2011; 11: 9-22.
[23] Tirelli G., Zacchigna S., Biasotto M., Piovesana M.: Open questions and novel concepts in oral cancer surgery. Eur. Arch. Otorhinolaryngol., 2016; 273: 1975-1985.

[24] Andratschke M., Schmitz S., Hagedorn H., Nerlich A.: Cytological and immunocytological monitoring of oropharyngeal dysplasia and squamous cell carcinomas. Anticancer Res., 2015; 35: 65176520 .

[25] Morrison L.F., Hopp E.S., Wu R.: II Diagnosis of malignancy of the nasopharynx. cytological studies by the smear technic. Ann. Otol. Rhinol. Laryngol., 1949; 58: 18-32.

[26] Friedmann I.: Exfoliative cytology as an aid in the diagnosis of tumours of the throat, nose and ear. J. Laryngol. Otol., 1951; 65: $1-9$.

[27] Frable W.F., Frable M.A.: Cytologic diagnosis of carcinoma of the larynx by direct smear. Acta Cytol. 1968; 12: 318-324.

[28] Glennie H.R., Gilbert J.G., Melcher D.H., Linehan J., Wadsworth P.V.: The place of cytology in laryngeal diagnosis. Clin. Otolaryngol. Allied Sci., 1976; 1: 131-136.

[29] Lundgren J., Olofsson J., Hellquist H.B., Strandh J.: Exfoliative cytology in laryngology: Comparison of cytologic and histologic diagnoses in 350 microlaryngoscopic examinations - a prospective study. Cancer, 1981; 47: 1336-1343.

[30] Waloryszak B., Makowska W.: The significance of the cytological evaluation in the early diagnosis of the laryngeal carcinoma. Otolaryngol. Pol., 1994; 48: 331-334.

[31] Malamou-Mitsi V.D., Assimakopoulos D.A., Goussia A., Pappa L., Skevas A.T., Agnantis N.J.: Contribution of exfoliative cytology to the diagnosis of laryngeal lesions. Acta Cytol., 2000; 44: 993-999.

[32] Ustundag E., Kaur A.C., Boyaci Z., Keskin G., Aydin O.: Combined use of histopathology with touch smear cytology in biopsies of the larynx. Eur. Arch. Otorhinolaryngol., 2006; 263: 866-871.

[33] Gugatschka M., Kiesler K., Beham A., Rechenmacher J., Friedrich G.: Hyperplastic epithelial lesions of the vocal folds: Combined use of exfoliative cytology and laryngostroboscopy in differential diagnosis. Eur. Arch. Otorhinolaryngol., 2008; 265: 797-801.

[34] Chatziavramidis A., Tsinaslanidou Z., Valeri R., Konstantinidis I., Constantinidis J.: Brush cytology with immunocytochemical evaluation of VEGF expression versus biopsy in clinically precancerous laryngeal lesions: Can we diagnose laryngeal cancer only with brush cytology? Anal. Cell. Pathol., 2015; 2015: 256182.

[35] Ajayan P.V., Ramesh S., Jacob A.M.: Correlation between exfoliative cytology and histopathology in laryngeal cancers - A descriptive study. Int. J. Biomed. Adv. Res. 2017; 8: 195-198. 